\title{
New Evidence in the Pathogenesis of Celiac Disease and Type 1 Diabetes Mellitus: A Systematic Review
}

Jose Prieto ${ }^{1}$, Karan B. Singh ${ }^{1}$, Maduka C. Nnadozie ${ }^{2}$, Muhammad Abdal ${ }^{3}$, Niki Shrestha ${ }^{2}$, Rose Anne M. Abe $^{2}$, Anum Masroor ${ }^{4,5}, 6$, Arseni Khorochkov ${ }^{1}$, Lubna Mohammed ${ }^{1}$

1. Internal Medicine, California Institute of Behavioral Neurosciences \& Psychology, Fairfield, USA 2. Research, California Institute of Behavioral Neurosciences \& Psychology, Fairfield, USA 3. Emergency Medicine, California Institute of Behavioral Neurosciences \& Psychology, Fairfield, USA 4. Psychiatry, California Institute of Behavioral Neurosciences \& Psychology, Fairfield, USA 5. Psychiatry, Psychiatric Care Associates, Englewood, USA 6. Medicine, Khyber Medical College, Peshawar, PAK

Corresponding author: Jose Prieto, joselu-as@hotmail.com

\begin{abstract}
Celiac disease (CD) and type 1 diabetes mellitus (T1DM) are autoimmune diseases that coexist frequently. These illnesses share a common genetic background. This study aims to review the different pathophysiologic mechanisms that have been studied about the coexistence of CD and T1DM, to contrast them, and to summarize their specific role in these autoimmune diseases. We conducted a systematic review following the Preferred Reporting Items for Systematic reviews and Meta-Analyses (PRISMA) checklist and used the Medical Subject Headings (MeSH) search strategy to obtain relevant articles. We found 585 papers which were reduced to 355 after removing duplicates. Later, the filters and inclusion/exclusion criteria were applied which ended the search with 78 articles. Finally, we reviewed the articles that contained information about the pathogenesis of $\mathrm{CD}$ and T1DM, their coexistence, and how the pathogenesis impacts clinical outcomes. The reviewed studies strongly conclude that the presence of human leukocyte antigen (HLA) genes DQ2 and DQ8 are high-risk for developing the coexistence of CD and T1DM. We found that killer immunoglobulin-like receptor (KIR) genes, enterovirus infection in gut cells, and gut microbiota dysbiosis with the predominance of Bacteroides spp. also play a role in the pathogenesis and development of symptoms of $\mathrm{CD}$ in patients with the previous diagnosis of T1DM. CD4+ and CD8+ cell levels vary among patients and studies, consequently, more study on this topic is needed.
\end{abstract}

Received 06/19/2021 Review began 07/07/2021 Review ended 07/18/2021 Published 07/29/2021

○ Copyright 2021 Prieto et al. This is an open access article distributed under the terms of the Creative Commons Attribution License CC-BY 4.0., which permits unrestricted use, distribution, and reproduction in any medium, provided the original author and source are credited.
Categories: Endocrinology/Diabetes/Metabolism, Allergy/Immunology, Gastroenterology

Keywords: celiac disease, type 1 diabetes mellitus (t1dm), human leukocyte antigen (hla), microbiota, autoimmune, immune profile

\section{Introduction And Background}

Celiac disease (CD) and type 1 diabetes mellitus (T1DM) are autoimmune diseases that affect approximately $1 \%$ and $0.5 \%$ of the general population worldwide, respectively [1]. They coexist in around $4-5 \%$ of the cases with T1DM occurring usually some years before CD [2]. CD is a disorder characterized by a hypersensitivity to gluten, a protein found in wheat, rye, barley, and others. When gluten is ingested, it causes a variety of gastrointestinal symptoms, but the most concerning is the malabsorptive syndrome produced principally by tissue transglutaminase antibodies (tTG), an IgA-mediated response [3]. Otherwise, T1DM is a classic autoimmune disorder in which there occurs an IgG-mediated pancreatic B-cell destruction by autoantibodies (glutamic acid decarboxylase antibodies - GAD) and later insulin deficiency with rising blood glucose [4].

The importance of this coexistence lies in that the patients have to deal with the symptomatology of both diseases. Usually, first, the polyuria; polydipsia; polyphagia; weight loss, all classic symptoms of diabetes, and then, the unknown development of $\mathrm{CD}$ and all the variety of symptoms of malabsorption and its complications. Also, the patients and their families have to manage and control their treatments with insulin dosing and a gluten-free diet, which sometimes are very troublesome [5].

It is well known that these illnesses share a common genetic background that predisposes individuals to develop them. The presence of human leukocyte antigen (HLA) type II alleles: HLA-DQ2 and HLA-DQ8, confer high risk to develop these diseases, this has been demonstrated with their presence in $98 \%$ of patients with CD and 95\% of patients with T1DM [6]. However, there is rising evidence suggesting that there are some other genetic, immunological, environmental, viral exposure, and dietary factors that may contribute some importance to the pathogenesis and coexistence of these diseases in the population [7]. Additionally, studies suggest that low expression or absence of regulatory T cells, helper T cells, certain cytokines, chemokines, and other inflammatory mediators, puts individuals at risk of developing CD and T1DM $[8,9]$. 


\section{Cureus}

Even though the recent discoveries about the different pathogenic and pathophysiologic mechanisms of CD and T1DM are done in low sample studies and few places in the world, they are completely reproducible to the general population and reliable in statistic terms. That is why the research on this topic is increasing and new mechanisms are being revealed to better understand these diseases.

We aim to review the different pathophysiologic mechanisms that have been studied about the coexistence of $\mathrm{CD}$ and T1DM, to contrast them and summarize their specific roles in these autoimmune diseases.

\section{Methods}

We conducted a systematic review following the Preferred Reporting Items for Systematic reviews and MetaAnalyses (PRISMA) checklist. We searched articles published on PubMed until May 3, 2021. We applied the Medical Subject Headings (MeSH) search strategy to obtain related articles, and the following keywords were included:

- Celiac disease

- Type 1 Diabetes Mellitus

- Celiac disease and Type 1 Diabetes Mellitus

Table 1 summarizes the strategy we used for MeSH terms.

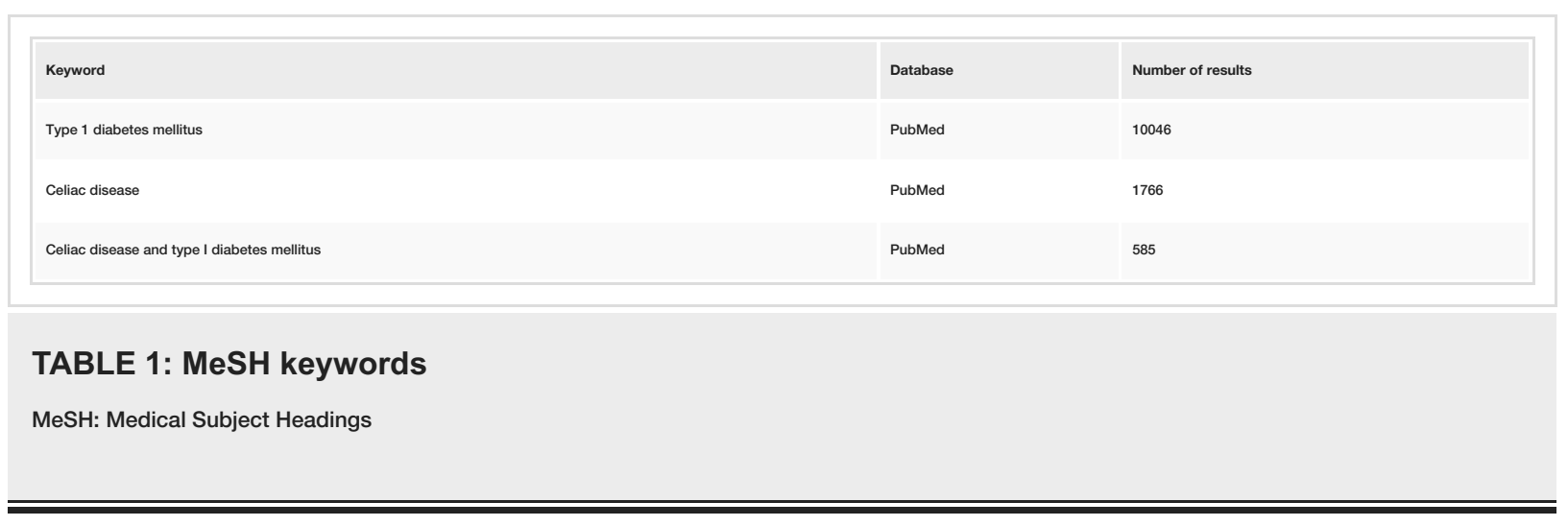

\section{Results}

After using the MeSH search strategy to search for interesting articles, we found 585 papers which were then reduced to 355 after removing the duplicates. Later, the filters and inclusion/exclusion criteria were applied which ended the search with 78 articles. The titles of 78 articles were screened to identify possible relevant articles for this research. Once we finished identifying relevant papers, we screened their titles again, including the abstracts, and full text of pertinent studies.

It is important to mention that two of the authors separately reviewed the quality of the studied papers by applying different quality assessment tools, depending on the type of article, including "Newcastle-Ottawa tool" for non-randomized controlled trials and observational studies, "PRISMA checklist" for systematic reviews, and "Scale for the Assessment of Narrative Review Articles (SANRA) checklist" for literature reviews. After this quality assessment, one article was excluded leaving 14 papers to review. The entire review was done scientifically and within ethical boundaries. All these processes are summarized in Figure 1 . 


\section{Cureus}
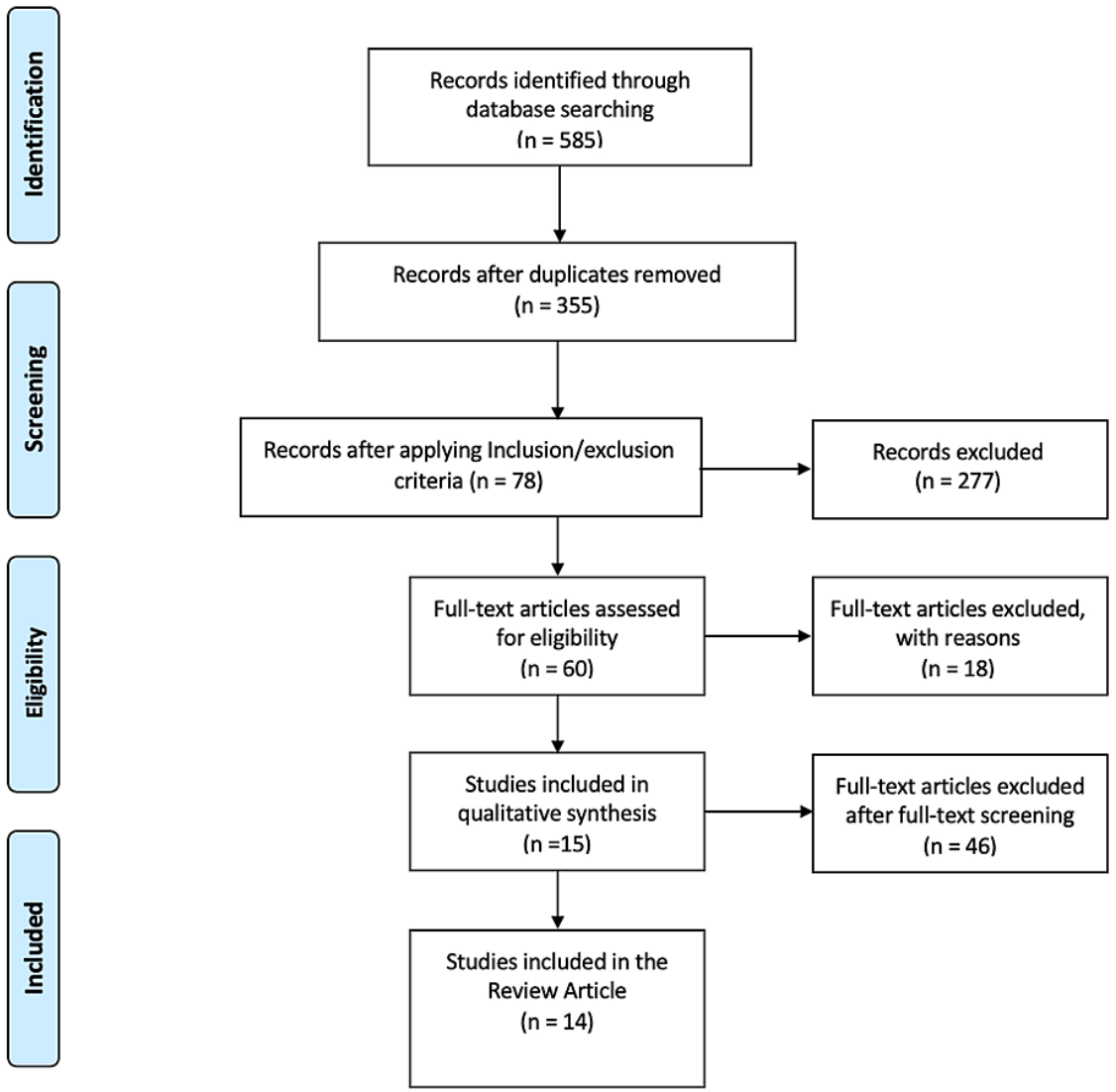

FIGURE 1: PRISMA flowchart

PRISMA: preferred reporting items for systematic reviews and meta-analyses

\section{Inclusion criteria}

We have included articles written in English in the last 10 years, done in humans. We focused on the main purpose of this research which is similarities in the pathogenesis of CD and T1DM in patients of any age and sex. All types of studies were included. All the articles included were peer-reviewed.

\section{Exclusion criteria}

Articles written in any language other than English, research done in animal models, grey literature, and published before May 3, 2011 were excluded.

\section{Review}

\section{Discussion}

During recent years, multiple studies have demonstrated the existing relation between T1DM and $\mathrm{CD}$. Various mechanisms and factors have been studied and included in these discussions, which we evaluate in this systematic review. The most established and accepted conclusion is that both diseases share a common genetic background, which is principally determined by HLA genes DQ2 and DQ8 [10].

\section{Genetics and immunology}

The importance of the association between these two diseases can be reflected in that in some countries the genotyping of HLA high-risk genes is mandatory if a patient is diagnosed with T1DM. While in other countries the coexistence of $\mathrm{CD}$ with T1DM has been so well established that when a patient is diagnosed with T1DM, it is not necessary to do more tests, given the high positive predictive values of these tests [11]. These studies have also shown that it is far more common for patients with T1DM to develop CD later in life than the opposite way [12]. Moreover, a big proportion of asymptomatic patients with a baseline diagnosis of T1DM also tests positive for anti-tissue transglutaminase antibodies, and its levels correlate with the degree of villous atrophy [13]. 
Given the importance of the association of these two diseases, some studies have been conducted to inquire into the mechanisms of this correlation and to better comprehend their behaviors. Likewise, it would help in the development of new ways to treat these sicknesses and improve the quality of life of the patients. In Table 2, we summarize the reviewed studies with the different mechanisms proposed to play a role in the correlation of T1DM and CD.

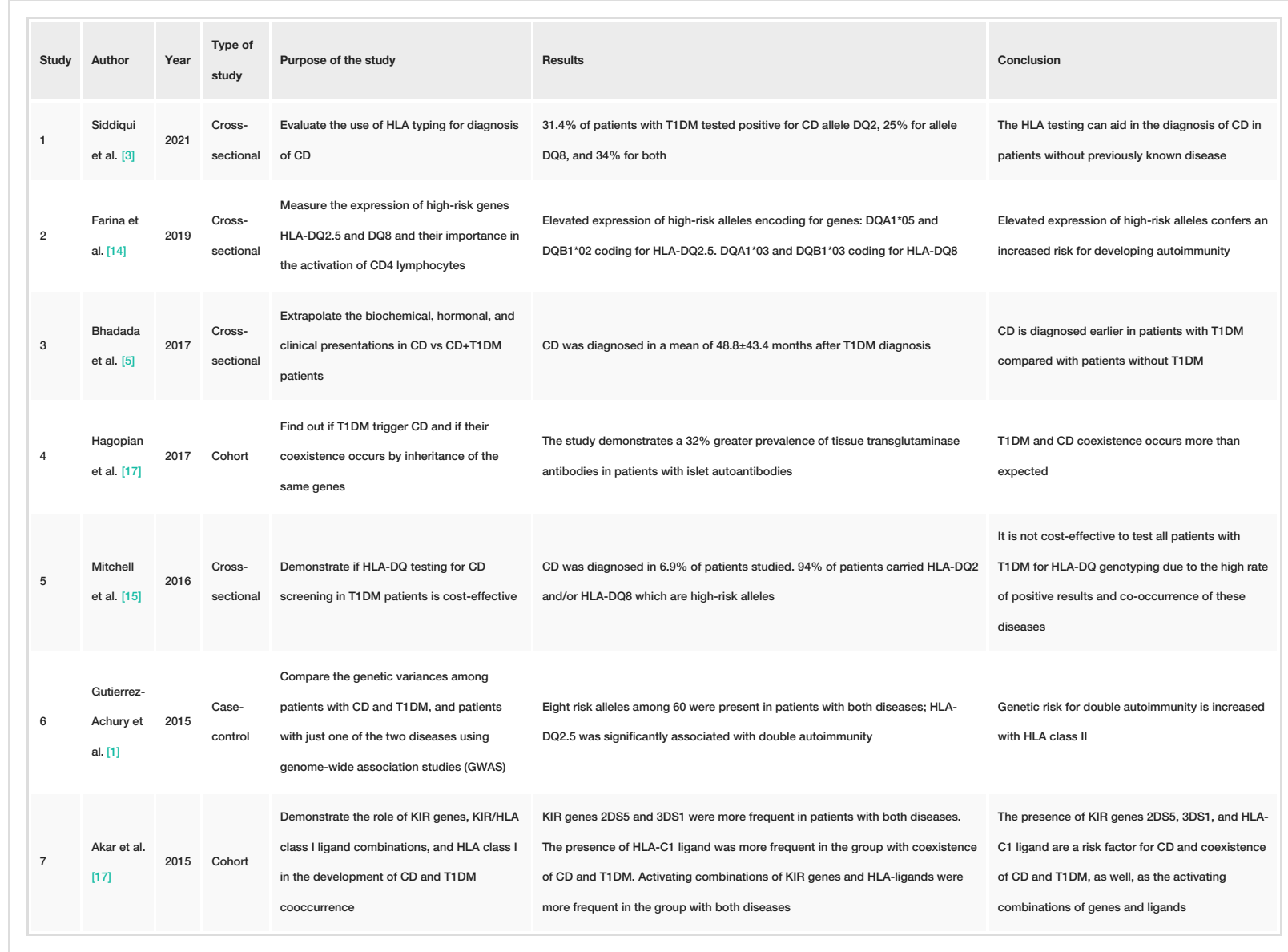

TABLE 2: Genetic and immunologic mechanisms in the correlation of type 1 diabetes and celiac disease

CD: celiac disease; HLA: human leukocyte antigen; KIR: killer immunoglobulin-like receptor; T1DM: type 1 diabetes mellitus

As seen in Table 2, all the reviewed studies showed an association between T1DM and CD, a fact we knew from previous research. But the new discoveries in pathogenesis and genetics are helping us to answer the previously unknown facts. We knew that HLA-DQ2.5 and DQ8 were the higher risk genes for developing both diseases, but now we know that the alleles DQA $1 * 05$ and DQB $1 * 02$ code for HLA-DQ2.5 and DQA 1 *03 and DQB1*03 code for HLA-DQ8 [14]. And thanks to the identification of the exact location of these alleles, we can use the HLA testing in high-risk patients to diagnose coexistence in asymptomatic patients [3]. However, due to the high percentage of positive alleles in T1DM patients, it is not recommended to test all patients because it is not cost-effective [16].

Akar et al. demonstrated a positive correlation between activating killer immunoglobulin-like receptor (KIR) genes, HLA class I ligand, and KIR/HLA class I ligand combinations, in patients with CD alone and in patients with T1DM and CD. Activating and inhibitory KIR genes control the activity of natural killer (NK) cells, which play a role in the pathogenesis of CD [16]. Their results are similar to other studies of the same class that show the impact of KIR genes in the development of CD [18]. This finding can be important in the elaboration of new strategies for the treatment of CD, especially in patients with baseline T1DM.

\section{Microbiota}

One of the newest advances in understanding the coexistence of CD and T1DM is the dysbiosis existing in patients with both diseases. The term "dysbiosis" is used to refer to an imbalance in normal gut microbiota which may cause different symptoms [19]. Patients with the coexistence of both diseases have similar bacterial gut composition, comparing them to patients with T1DM only, which can be attributed to the fact 
that patients develop T1DM before CD [20]. Also, the high levels of certain bacteria like Bacteroides and low levels of others like Coprococcus and Prevotella may play a role in the development of the classic symptomatology of $\mathrm{CD}$, given that some bacteria use gluten as a fuel and the products of this metabolism may be the cause of the classic symptoms [7, 20,21]. However, more studies are needed to clarify these statements. Table 3 contains the evidence found about the changes in microbiota in patients with the illnesses in study.

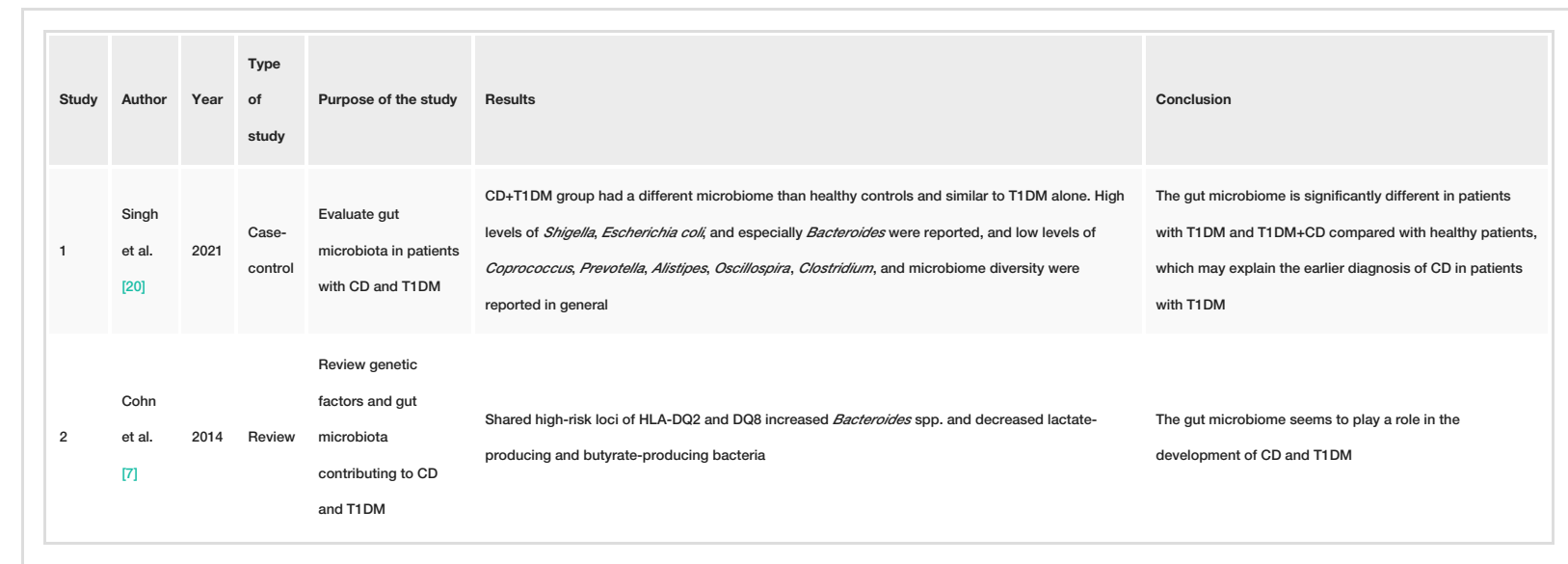

TABLE 3: Microbiota in patients with celiac disease and type 1 diabetes mellitus coexistence CD: celiac disease; HLA: human leukocyte antigen; T1DM: type 1 diabetes mellitus

\section{Immune profile and environmental factors}

Another discovery in pathogenesis is that patients with $\mathrm{CD}$ and T1DM have altered levels of cytokines, metalloproteinases, chemokines, acute phase proteins, and antibodies, compared with healthy controls. Tiberti et al. showed that patients with a previous diagnosis of T1DM had a low immune response at the time of diagnosis of $\mathrm{CD}$ years later. This finding may explain the difference in diagnosis time among different patients [22]. On the other hand, Tompa et al. found decreased levels of certain substances like interleukin-22 (IL-22), macrophage inhibitor potentiator protein (MIP-1 $\alpha$ ), monocyte chemoattractant protein-1 (MCP-1), acute phase proteins, procalcitonin, fibrinogen, adipocytokine visfatin, and matrix metalloproteinases (MMP-2). This finding may confer a protective role in the development of CD in patients with T1DM [8]. Another research like Guan et al. studied the levels of specific cytokines like MCP-1 and showed low levels of it in patients with T1DM compared with healthy controls [23]. Similarly, El Samahi et al. studied the levels of intracellular enzyme visfatin in patients with T1DM, showing lower levels compared with healthy controls [24]. All of this information demonstrates that the low levels of cytokine, enzyme, proteins, etc. may play a protective role in the pathogenesis of T1DM and CD. However, as suggested in the papers previously reviewed, studies with greater samples are needed to have better conclusions.

Opposite to the previous discussion, Vorobjova et al. found increased levels of certain cytokines such as IL17F, interferon-gamma-induced protein (IP-10), soluble tumor factor receptor 2 (sTNFRII), MCP-1, and granulocyte-macrophage colony-stimulating factor (GM-CSF), which correlates with the level of inflammation and enterovirus infection in the gut cells of patients with CD and T1DM [25]. This finding is also present in the study of Goodwin, who, in his review, also said that the presence of enterovirus in gut cells had increased the risk and was present in patients with coexistence of T1DM and CD [2]. This can be corroborated with the results of the TEDDY study (The Environmental Determinants of Diabetes in the Young) which was conducted with a sample of 8676 children, where they strongly demonstrated that the enterovirus infection predisposes to the development of $\mathrm{CD}$ in diabetic patients [26].

Another fact to mention is that Åkesson et al. had shown an elevated percentage of terminally differentiated helper T cells $\mathrm{CD} 4+$ and a decreased amount of effector memory cytotoxic $\mathrm{T}$ cells, early differentiated and late differentiated CD8+ [9]. However, the authors concluded that their findings were not conclusive due to the differences with other studies in which the levels of CD4+ and CD8+ cells were positively correlated with autoimmunity and reactivity to gluten in $\mathrm{CD}$ [27]. In Table 4, we summarize the findings of the studies previously mentioned. 


\section{Cureus}

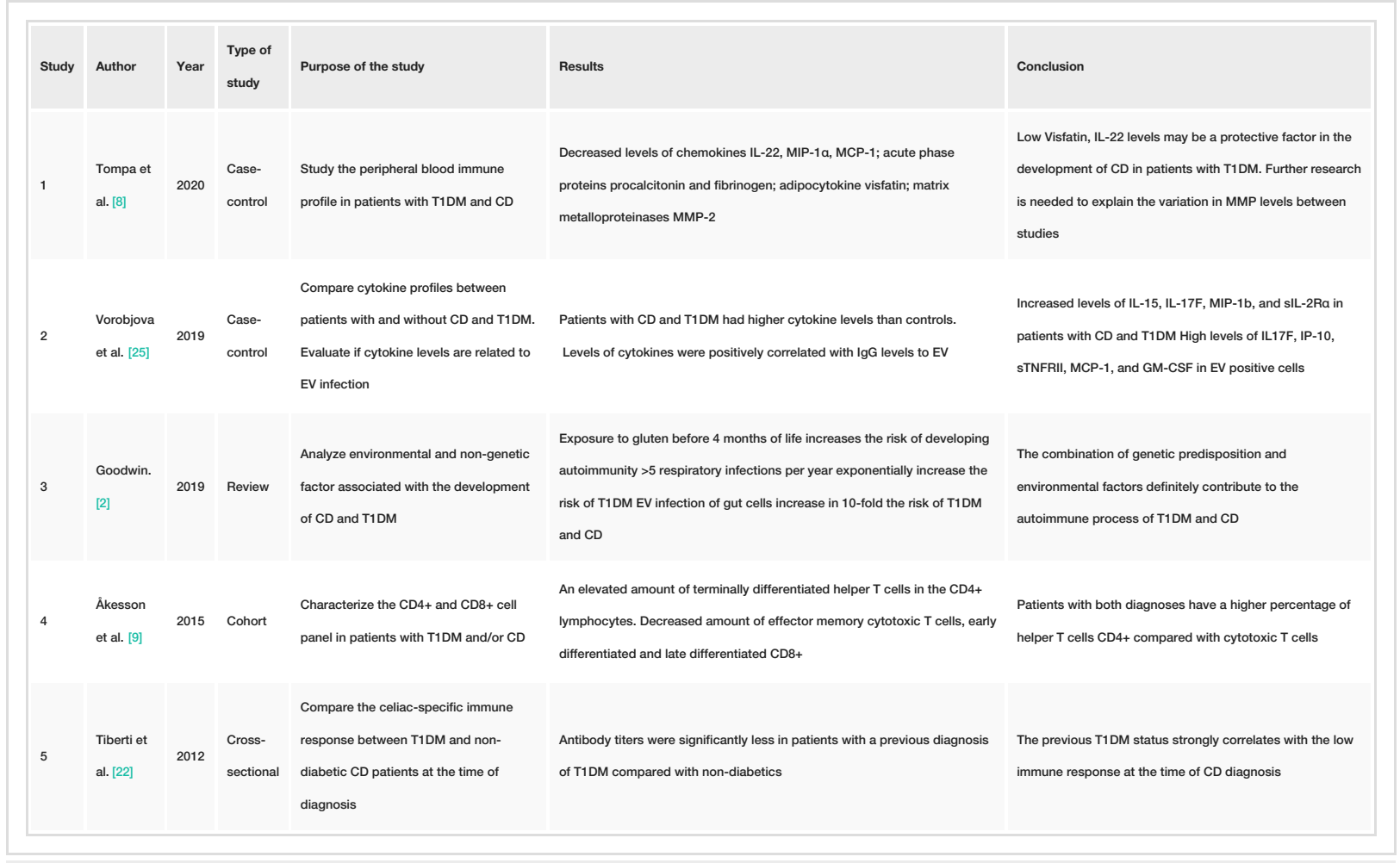

TABLE 4: Immune profile and environmental factors in patients with celiac disease and type 1 diabetes coexistence

CD: celiac disease; GM-CSF: granulocyte-macrophage colony-stimulating factor; IL: interleukin; IP-10: interferon gamma-induced protein 10; MCP1: monocyte chemoattractant protein 1; MIP-1a: macrophage inhibitor potentiator protein 1 alpha; MIP-1b: macrophage inhibitor potentiator protein 1 beta; sIL-2Ra: soluble interleukin 2 receptor alpha; sTNFRII: soluble tumor factor receptor II; T1DM: type 1 diabetes mellitus; EV: enterovirus

\section{Limitations}

During this review, we found some limitations. First, the information obtained was from one database only (PubMed). Second, various types of studies were included, including cohorts, cross-sectionals, reviews, and case controls; therefore, the differences in samples and statistic methods used in the different studies make our study prone to bias. Third, the majority of papers included were done with small samples, and in different stages of the diseases. Thus, studies with greater samples are needed in order to get more conclusive results.

\section{Conclusions}

We aimed to review the different pathogenic factors that are proposed to play a role in the coexistence of $C D$ and T1DM, which is important to understand them and to potentially use this information for the improvement of new treatment strategies. To conclude, we can say that HLA-DQ2.5 and HLA-DQ8 are the higher risk genes to develop coexistence of these diseases, basically its presence tells us why these two appear together in a great amount of cases; also, the testing of these genes can be beneficial in the diagnosis of $\mathrm{CD}$ and T1DM depending on the clinical condition of every specific patient. Additionally, that KIR genes controlling NK cell activity have a crucial role in the development of clinical symptoms of $\mathrm{CD}$ in patients with previous T1DM diagnosis. One new discovery that is gaining terrain in this field is that dysbiosis in gut microbiota has also played a very important role in the development of the symptoms of CD. The reviewed studies emphasized the elevated presence of Bacteroides spp. and gluten-using bacteria. In the same way, the evidence shows a clear role of enterovirus infection in gut cells and degree of inflammation. Finally, we have seen an imbalance in the immune profile of patients with the coexistence of CD and T1DM, especially referring to the levels of $\mathrm{CD} 4+$ and $\mathrm{CD} 8+$ cells. All these factors can give us a brief overview of why these illnesses vary so widely among individuals. However, due to the discrepancy between studies on these topics, we suggest additional research with greater samples and statistically similar control groups to obtain definitive results.

\section{Additional Information}

\section{Disclosures}

Conflicts of interest: In compliance with the ICMJE uniform disclosure form, all authors declare the 
following: Payment/services info: All authors have declared that no financial support was received from any organization for the submitted work. Financial relationships: All authors have declared that they have no financial relationships at present or within the previous three years with any organizations that might have an interest in the submitted work. Other relationships: All authors have declared that there are no other relationships or activities that could appear to have influenced the submitted work.

\section{Acknowledgements}

I would like to thank all my colleagues with whom I had the pleasure to work during this project, my parents and family for their unconditional support every time, and special thanks to my dear wife, Carla, for her tireless and infinite support and love in every step of my life.

\section{References}

1. Gutierrez-Achury J, Romanos J, Bakker SF, et al.: Contrasting the genetic background of type 1 diabetes and celiac disease autoimmunity. Diabetes Care. 2015, 38 Suppl 2:S37-44. 10.2337/dcs15-2007

2. Goodwin G: Type 1 diabetes mellitus and celiac disease: distinct autoimmune disorders that share common pathogenic mechanisms. Horm Res Paediatr. 2019, 92:285-92. 10.1159/000503142

3. Siddiqui K, Uqaili AA, Rafiq M, Bhutto MA: Human leukocyte antigen (HLA)-DQ2 and -DQ8 haplotypes in celiac, celiac with type 1 diabetic, and celiac suspected pediatric cases. Medicine (Baltimore). 2021, 100:e24954. 10.1097/MD.0000000000024954

4. Derrou S, El Guendouz F, Benabdelfedil Y, Chakri I, Ouleghzal H, Safi S: The profile of autoimmunity in type 1 diabetes patients. Ann Afr Med. 2021, 20:19-23. 10.4103/aam.aam_8_20

5. Bhadada SK, Rastogi A, Agarwal A, Kochhar R, Kochhar R, Bhansali A: Comparative study of clinical features of patients with celiac disease \& those with concurrent celiac disease \& type 1 diabetes mellitus. Indian J Med Res. 2017, 145:334-8. 10.4103/ijmr.IJMR_666_14

6. Kaur N, Bhadada SK, Minz RW, Dayal D, Kochhar R: Interplay between type 1 diabetes mellitus and celiac disease: implications in treatment. Dig Dis. 2018, 36:399-408. 10.1159/000488670

7. Cohn A, Sofia AM, Kupfer SS: Type 1 diabetes and celiac disease: clinical overlap and new insights into disease pathogenesis. Curr Diabetes Rep. 2014, 14:517. 10.1007/s11892-014-0517-X

8. Tompa A, Åkesson K, Karlsson S, Faresjö M: Suppressed immune profile in children with combined type 1 diabetes and celiac disease. Clin Exp Immunol. 2020, 201:244-57. 10.1111/cei.13454

9. Åkesson K, Tompa A, Rydén A, Faresjö M: Low expression of CD39(+) /CD45RA(+) on regulatory T cells (Treg ) cells in type 1 diabetic children in contrast to high expression of CD101(+)/CD129(+) on Treg cells in children with coeliac disease. Clin Exp Immunol. 2015, 180:70-82. 10.1111/cei.12559

10. Camarca ME, Mozzillo E, Nugnes R, et al.: Celiac disease in type 1 diabetes mellitus . Ital J Pediatr. 2012, 38:10. 10.1186/1824-7288-38-10

11. Deja G, Sikora D, Pyziak-Skupien A, Klenczar K, Deja R, Jarosz-Chobot P: The usefulness of genotyping of celiac disease-specific HLA among children with type 1 diabetes in various clinical situations. J Diabetes Res. 2020, 2020:1-8. 10.1155/2020/7869350

12. Elfström P, Sundström J, Ludvigsson JF: Systematic review with meta-analysis: associations between coeliac disease and type 1 diabetes. Aliment Pharmacol Ther. 2014, 40:1123-32. 10.1111/apt.12973

13. Kaur N, Minz RW, Bhadada SK, et al.: Role of anti-tissue transglutaminase IgA+IgG antibodies in detection of potential celiac disease in patients with type 1 diabetes. Indian J Med Res. 2019, 149:18-25. 10.4103/ijmr.IJMR 113616

14. Farina F, Picascia S, Pisapia L, et al.: HLA-DQA1 and HLA-DQB1 alleles, conferring susceptibility to celiac disease and type 1 diabetes, are more expressed than non-predisposing alleles and are coordinately regulated. Cells. 2019, 8:751. 10.3390/cells8070751

15. Mitchell RT, Sun A, Mayo A, Forgan M, Comrie A, Gillett PM: Coeliac screening in a Scottish cohort of children with type 1 diabetes mellitus: is DQ typing the way forward?. Arch Dis Child. 2016, 101:230-3. 10.1136/archdischild-2015-309754

16. Akar HH, Patiroglu T, Sevinc E, Aslan D, Okdemir D, Kurtoglu S: Contribution of KIR genes, HLA class I ligands, and KIR/HLA class I ligand combinations on the genetic predisposition to celiac disease and coexisting celiac disease and type 1 diabetes mellitus. Rev Esp Enferm Dig. 2015, 107:547-53. 10.17235/reed.2015.3817/2015

17. Hagopian W, Lee HS, Liu E, et al.: Co-occurrence of type 1 diabetes and celiac disease autoimmunity . Pediatrics. 2017, 140:e20171305. 10.1542/peds.2017-1305

18. Smigoc Schweiger D, Mendez A, Kunilo Jamnik S, et al.: Genetic risk for co-occurrence of type 1 diabetes and celiac disease is modified by HLA-C and killer immunoglobulin-like receptors. Tissue Antigens. 2014, 84:471-8. 10.1111/tan.12450

19. Caio G, Volta U, Sapone A, Leffler DA, De Giorgio R, Catassi C, Fasano A: Celiac disease: a comprehensive current review. BMC Med. 2019, 17:142. 10.1186/s12916-019-1380-Z

20. Singh P, Rawat A, Al-Jarrah B, et al.: Distinctive microbial signatures and gut-brain crosstalk in pediatric patients with coeliac disease and type 1 diabetes mellitus. Int J Mol Sci. 2021, 22:1511. 10.3390/ijms22041511

21. Olivares M, Benítez-Páez A, de Palma G, et al.: Increased prevalence of pathogenic bacteria in the gut microbiota of infants at risk of developing celiac disease: the PROFICEL study. Gut Microbes. 2018, 9:551-8. 10.1080/19490976.2018.1451276

22. Tiberti C, Panimolle F, Bonamico M, et al.: IgA anti-transglutaminase autoantibodies at type 1 diabetes onset are less frequent in adult patients and are associated with a general celiac-specific lower immune response in comparison with nondiabetic celiac patients at diagnosis. Diabetes Care. 2012, 35:2083-5. 10.2337/dc11-2171

23. Guan R, Purohit S, Wang H, et al.: Chemokine (C-C motif) ligand 2 (CCL2) in sera of patients with type 1 


\section{Cureus}

diabetes and diabetic complications. PLoS One. 2011, 6:e17822. 10.1371/journal.pone.0017822

24. El Samahi MH, Ismail NA, Matter RM, Selim A, Ibrahim AA, Nabih W: Study of visfatin level in type 1 diabetic children and adolescents. Open Access Maced J Med Sci. 2017, 5:299-304. 10.3889/oamjms.2017.065

25. Vorobjova T, Tagoma A, Oras A, et al.: Celiac disease in children, particularly with accompanying type 1 diabetes, is characterized by substantial changes in the blood cytokine balance, which may reflect inflammatory processes in the small intestinal mucosa. J Immunol Res. 2019, 2019:1-17.

$10.1155 / 2019 / 6179243$

26. Lindfors K, Lin J, Lee HS, et al.: Metagenomics of the faecal virome indicate a cumulative effect of enterovirus and gluten amount on the risk of coeliac disease autoimmunity in genetically at risk children: the TEDDY study. Gut. 2020, 69:1416-22. 10.1136/gutjnl-2019-319809

27. Jabri B, Sollid LM: T Cells in Celiac Disease. J Immunol. 2017, 198:3005-14. 10.4049/jimmunol.1601693 